\title{
MAC LANE METHOD FOR DETERMINATION OF EXTENSIONS OF FINITE GROUPS. II. AN EXAMPLE FOR THE DIHEDRAL GROUP $D_{2}$
}

\author{
T. LULEK
}

Institute of Physics, A. Mickiewicz Univcrsily, Matejki 48/49, 60-769 Poznani, Poland

and R. Chatterjee

Department of Pliysics and Astronomy, University of Calgary

Calgary, Alberta T2N 1N4, Canada

(Received May 28, 1992)

\begin{abstract}
The Mac Lane method of classification and construction of all extensions of a group $Q$ by an Abelian group $T$ is demonstrated on the case $Q=D_{2}, T=C_{2}$. Constructions involving free groups and operator homomorphisms are performed in detail, and the complete list of resulting extensions is given. It is shown that there are 8 classes of gauge equivalency, and they fall into 4 classes of isomorphism. The role of gauge transformations is pointed out. Physical contexts of various constructions are reviewed. A comparison with the direct cohomology definitions is performed.
\end{abstract}

PACS numbers: 02.20. $+\mathrm{b}, 02.40 .+\mathrm{m}, 03.20 .+\mathrm{i}, 11.30 . \mathrm{E}_{\mathrm{r}}$

\section{Introduction}

In part I [1] of this review the general Mac Lane method of construction of equivalency classes of extensions of a finite group $Q$ by an Abelian group $T$ under a given action $\Delta: Q \rightarrow \Lambda$ ut $T$ has been presented in detail, and its relevance in physics has been pointed out within the context of crystallograpliy and gauge fields. Here we intend to demonstrate this general method on a specific cxample of the simplest dihedral group

$$
Q=D_{2}=\left\{E, u_{x}, u_{y}, u_{z}\right\},
$$

where $E$ denotes the unit element, and $u_{x}, u_{y}, u_{z}$ are twofold axes coinciding with a Cartesia coordinate system. In the first stage we assume that $T$ is an arbitrary finite $\Lambda$ belian group, and next we specify it as the two-element group

$$
T=C_{2}=\{E, \bar{E}\}
$$


with the non-trivial element denoted by $\vec{E}$. In particular, we are going to classify all non-equivalent extensions of $D_{2}$ by $C_{2}$, i.e. to determine the second cohomology group $H^{2}\left(D_{2}, C_{2}\right)$ (in this case the action $\Delta$ is trivial). This case is simple cnough to demonstrate in detail the most of the notions of the Mac Lane method presented in [1], and to provide some physical interpretation to various algebraic and combinatorial constructions. This work is notivated by our belief that such an approach can shed some new light into the fascinating problem of combination ways of geometric and gauge symmetries, and related phenomena like quantum IIall effect [2], Berry phases [3-5], topological invariants [6], flux quantization [7], etc., by exposing the possibilities of construction of extensions of some groups already known in crystallography.

Clearly, the group $D_{2}$ has a crystallographic interpretation as a point group, consisting of three mutually perpendicular twofold axes, coinciding with axes of a Cartesian coordinate system in an Euclidean three-dimensional space. In quantum field theorics [10] it arises as the group of discrete space-time inversion symmetry (c.g. $u_{x}=P, u_{y}=T, u_{z}=P T$ with $P$ and $T$ being respectively parity and time reversal); in particular, it labels the four disjoint pieces of the Lorentz group. It is also isomorphic with the automorphism group for some cyclic groups $C_{N}$ (e.g. $N=8$, or $N=12$ ), and thus serves as the group of hidden symmetry of the recipe of Weyl [11], applied to a closed linear chain of $N$ atoms with an interpretation of fractal symmetry $([12,13])$.

In the following we use the notation introduced in [1].

\section{Free groups and their alphabets}

The Mac Lane method of construction of all extensions $G$ of the group $Q$ by the group $T$ uses the following cxact scquence

$$
0 \rightarrow R \stackrel{i}{\rightarrow} F \stackrel{M}{\rightarrow} Q \rightarrow 1
$$

as a covering prototype of the exact sequence

$$
0 \rightarrow T \stackrel{\kappa}{\rightarrow} G \stackrel{\omega}{\rightarrow} Q \rightarrow 1
$$

which defines the extension $G$. IIere $i$ and $\kappa$ are injection monomorphisms, $M$ and $\omega$ are epimorphisms, $F$ is the free group, gencrated freely from a set $A$ of generators of the group $Q$, and $R$ is the kernel of the monomorphism $M$ (cf. [1] for more detail).

In the case $Q=D_{2}$ we choose the set

$$
A=\left\{u_{x}, u_{y}\right\}
$$

as generators of $D_{2}$, and thus we write down the alphabet $X$ of the free group $F$ as

with

$$
X=\left\{x_{1}, x_{2}\right\},
$$

$$
M\left(x_{1}\right)=u_{x}, \quad M\left(x_{2}\right)=u_{y} .
$$

The decomposition of the group $F$ into right cosets with respect to the subgroup $R$, i.e.

$$
F=R \cup R x_{1} \cup R x_{2} \cup R x_{1} x_{2},
$$


corresponds to the choice of the Schreier set $S$ as

$$
S=\left\{e_{F}, x_{1}, x_{2}, x_{1} x_{2}\right\} .
$$

The Schreier set $S$ determines the factor system $\rho: Q \times Q \rightarrow R$ for the covering exact sequence (3) by means of the formula

$$
f_{q_{1}} f_{q_{2}}=\rho\left(q_{1}, q_{2}\right) f_{q_{1} q_{2}}, \quad\left(q_{1}, q_{2}\right) \in Q^{2}
$$

with the right coset representatives $\int_{q}$ belonging to $S, q \in Q$. The results are listed in Table I. Clearly, entries $\rho\left(q_{1}, q_{2}\right)$ of the factor system $\rho$ belong to the subgroup $R \triangleleft F$, but they are expressed in Table I in terms of the alphabet $X$ of the group $F$.

\section{TABLE I}

The factor system $\rho: Q \times Q \rightarrow R$, expressed in terms of the alphabet $X$ of the group $F . e_{F}$ is the unit element of the group $F$. Observe that $\left\{x_{1}, x_{2}\right\}=$ $X \subset S=\left\{e_{F}, x_{1}, x_{2}, x_{1} x_{2}\right\}$, and thus all non-trivial entries of the second and third column constitute the alphabet $Y$ of the subgroup $R \triangleleft F$.

\begin{tabular}{c|c|c|c|c}
\hline \hline & $e_{F}$ & $x_{1}$ & $x_{2}$ & $x_{1} x_{2}$ \\
\hline$e_{F}$ & $e_{F}$ & $e_{F}$ & $e_{F}$ & $e_{F}$ \\
$x_{1}$ & $e_{F}$ & $x_{1}^{2}$ & $e_{F}$ & $x_{1}^{2}$ \\
$x_{2}$ & $e_{F}$ & $x_{2} x_{1} x_{2}^{-1} x_{1}^{-1}$ & $x_{2}^{2}$ & $x_{2} x_{1} x_{2} x_{1}^{-1}$ \\
$x_{1} x_{2}$ & $e_{F}$ & $x_{1} x_{2} x_{1} x_{2}^{-1}$ & $x_{1} x_{2}^{2} x_{1}^{-1}$ & $x_{1} x_{2} x_{1} x_{2}$
\end{tabular}

We observe that the alphabet $X$ is a subset of the Schreier set $S$. This observation allows us to identify the alphabet $Y$ of the subgroup $R$ with the set of all non-trivial elements of the second and third columns of Table I since

$$
Y=\left\{\rho\left(q_{1}, q_{2}\right) \mid M\left(q_{1}\right) \in S, M\left(q_{2}\right) \in X\right\}
$$

(cf. Eqs. (58) and (79) of [1]). Thus we have

$$
\begin{gathered}
Y=\left\{y_{1}=x_{1}^{2}, y_{2}=x_{2}^{2}, y_{3}=x_{2} x_{1} x_{2}^{-1} x_{1}^{-1},\right. \\
\left.y_{4}=x_{1} x_{2} x_{1} x_{2}^{-1}, y_{5}=x_{1} x_{2}^{2} x_{1}^{-1}\right\} .
\end{gathered}
$$

The number of all letters in the alphabet $Y$ satisfies Eq. (61) of [1], which reads

$$
5=|Y|=1+(|X|-1)|Q|=1+(2-1) \cdot 4 \text {. }
$$

Using Eq. (78) of [1], one can readily express the factor system $\rho$ in terms of the alphabet $Y$. The results are given in Table II. In fact, Eq. (12) determines the monomorphism $i: R \rightarrow F$ of the exact sequence (3).

The free group $F$ acts on its subgroup $R$ by inner automorphisms. This action, denoted in [1] by $\Xi: F \rightarrow$ Aut $R$, is defined using both alphabets, $X$ and $Y$, as

$$
\Xi(x)=\left(\begin{array}{c}
y \\
x y x^{-1}
\end{array}\right), x \in X, y \in Y .
$$


TABLE II

The factor systcm $\rho: Q \times Q \rightarrow R$, expressed in terms of the alphabet $Y$ of the subgroup $R \triangleleft F$. Trivial entries $\rho(q, E)=\rho(E, q)=e_{F}, q \in D_{2}$, arc omitted.

\begin{tabular}{c|c|c|c}
\hline \hline & $u_{x}$ & $u_{y}$ & $u_{z}$ \\
\cline { 2 - 4 }$u_{x}$ & $y_{1}$ & $e_{F}$ & $y_{1}$ \\
$u_{y}$ & $y_{3}$ & $y_{2}$ & $y_{3} y_{5}$ \\
$u_{z}$ & $y_{4}$ & $y_{5}$ & $y_{4} y_{2}$
\end{tabular}

TABLE III

The action $\Xi: F \rightarrow$ Aut $R$ (cᄃ. Eq. (14)). Entries of the table are $x y x^{-1}$ written in the alphabet $Y$.

\begin{tabular}{c|c|c|c|c|c}
\hline \hline & $y_{1}$ & $y_{2}$ & $y_{3}$ & $y_{4}$ & $y_{5}$ \\
\cline { 2 - 6 }$x_{1}$ & $y_{1}$ & $y_{5}$ & $y_{4} y_{1}^{-1}$ & $y_{1} y_{3}$ & $y_{1} y_{2} y_{1}^{-1}$ \\
$x_{2}$ & $y_{3} y_{4}$ & $y_{2}$ & $y_{2} y_{5}^{-1} y_{3}^{-1}$ & $y_{3} y_{5} y_{1} y_{2}^{-1}$ & $y_{3} y_{5} y_{3}^{-1}$
\end{tabular}

Table III describes this action by expressing each $x y x^{-1}$ in terms of the alphabet $Y$. All the above results, i.e. the alphabet $Y$ of the group $R$ of all relations of the group $Q$, the factor system $\rho: Q \times Q \rightarrow R$, and the action $\Xi: F \rightarrow$ Aut $R$, are determined by the active group $Q$ of the exact sequence (4). Further steps of the Mac Lane method involve also the passive group $T$. They will be considered in the next chapters.

\section{Operator homomorphisms and two-cocycles}

Construction of the group $\operatorname{IIom}_{F}(R, T)$ of all operator homomorplisms from the kernel $R$ of the epimorphism $M: F \rightarrow Q$ to the group $T$ (which is the kernel of the epimorphism $\omega: G \rightarrow Q$ ) starts from the manifold

$$
T^{Y}=\{\varphi: Y \rightarrow T\} \cong \operatorname{Hom}(R, T)
$$

of all mappings from the alphabet $Y$ of the group $R$ into the group $T$. Clearly, each such mapping generates a single homomorphism, therefore, in particular,

$$
\left|\operatorname{IIom}\left(R, C_{2}\right)\right|=2^{5}=32 \text {. }
$$

In general, the set (15) is the main subject of combinatorial enumeration theory [14], and its members can be arranged into orbits of some permutation groups, acting on $T$ and $Y[15,16]$. IIere, the size of the manifold $C_{2}^{Y}$ is so small that we do not specify any classification of its elements.

We have to select from the manifold $T^{Y}$ the submanifold of all operator homomorphisms, i.e. such mappings which intertwine the actions $\Xi: F \rightarrow$ Aut $R$ and $\Delta: Q \rightarrow$ Aut $T$. They have to satisfy the conditions

$$
\varphi\left(x y x^{-1}\right)=M(x) \varphi(y), \quad x \in X, y \in Y
$$


TABLE IV

Conditions (17) for operator homomorphisms $\varphi: R \rightarrow T$. Alphabets $X$ and $Y$ classify respectively the columns and the rows of the table. The left-hand side of each entry is $\varphi\left(x y x^{-1}\right)$ (in additive notation for the group $T$ ), whereas the right-hand side is $M(x) \varphi(y)$ (for the trivial action $\Delta$ of $D_{2}$ in $T$ ).

\begin{tabular}{c|c|c}
\hline \hline & $x_{1}$ & $x_{2}$ \\
\hline$y_{1}$ & $\varphi\left(y_{1}\right)=\varphi\left(y_{1}\right)$ & $\varphi\left(y_{3}\right)+\varphi\left(y_{4}\right)=\varphi\left(y_{1}\right)$ \\
$y_{2}$ & $\varphi\left(y_{5}\right)=\varphi\left(y_{2}\right)$ & $\varphi\left(y_{2}\right)=\varphi\left(y_{2}\right)$ \\
$y_{3}$ & $\varphi\left(y_{4}\right)-\varphi\left(y_{1}\right)=\varphi\left(y_{3}\right)$ & $\varphi\left(y_{2}\right)-\varphi\left(y_{5}\right)-\varphi\left(y_{3}\right)=\varphi\left(y_{3}\right)$ \\
$y_{4}$ & $\varphi\left(y_{1}\right)+\varphi\left(y_{3}\right)=\varphi\left(y_{4}\right)$ & $\varphi\left(y_{1}\right)-\varphi\left(y_{2}\right)+\varphi\left(y_{3}\right)+\varphi\left(y_{5}\right)=\varphi\left(y_{4}\right)$ \\
$y_{5}$ & $\varphi\left(y_{2}\right)=\varphi\left(y_{5}\right)$ & $\varphi\left(y_{5}\right)=\varphi\left(y_{5}\right)$
\end{tabular}

(cf. Table III). We rewrite these conditions in Table IV, where we assume the trivial action of $Q$ on $T$. This tablc contains $|X| \cdot|Y|=2 \cdot 5=10$ conditions, labelled by pairs $(y, x), y \in Y, x \in X$. Clearly, not all these conditions are independent. Three of them, namcly $\left(y_{1}, x_{1}\right),\left(y_{2}, x_{2}\right)$, and $\left(y_{5}, x_{2}\right)$, are identities. It is convenient to specify the group $T$ before evaluating further conditions. We assume here the additive notation for the group $T=C_{2}$, i.e. we put 0 and 1 for $E$ and $\bar{E}$, respectively, with addition modulo 2 . Then the conditions $\left(y_{2}, x_{1}\right),\left(y_{5}, x_{1}\right)$, and $\left(y_{3}, x_{2}\right)$ yicld

$$
\varphi\left(y_{2}\right)=\varphi\left(y_{5}\right)
$$

$\left(y_{3}, x_{1}\right),\left(y_{4}, x_{1}\right)$ and $\left(y_{1}, x_{2}\right)$ yield

$$
\varphi\left(y_{1}\right)+\varphi\left(y_{3}\right)+\varphi\left(y_{4}\right)=0
$$

and the last condition $\left(y_{4}, x_{2}\right)$ depends arithmetically on (18) and (19). Thus the submanifold $\operatorname{IIom}_{F}(R, T)$ of $T^{Y}$ is gencrated by three independent variables, e.g. $y_{1}, y_{2}$, and $y_{3}$, so that

$$
\left|\operatorname{Iom}_{F}\left(R, C_{2}\right)\right|=2^{3}=8 .
$$

The list of all operator homomorphisms is given in Table $\mathrm{V}$.

Each operator homomorphism $\varphi \in \operatorname{Iom}_{\Gamma}\left(R, C_{2}\right)$ yields a two-cocycle $m$ : $Q \times Q \rightarrow C$, given by

$$
m\left(q_{1}, q_{2}\right)=\varphi\left(\rho\left(q_{1}, q_{2}\right)\right), \quad\left(q_{1}, q_{2}\right) \in Q^{2},
$$

where $\rho: Q \times Q \rightarrow R$ is given by Table II. These two-cocycles are listed in Table VI. 
TABLE V

The group $\operatorname{Iom}_{F}\left(R, C_{2}\right)$ of operator homomorphisms. In this case, the group is isomorphic with the second cohomology group $H^{2}\left(D_{2}, C_{2}\right)$.

\begin{tabular}{c|c|c|c|c|c}
\hline \hline & $y_{1}$ & $y_{2}$ & $y_{3}$ & $y_{4}$ & $y_{5}$ \\
\hline$\varphi_{1}$ & 0 & 0 & 0 & 0 & 0 \\
$\varphi_{2}$ & 0 & 0 & 1 & 1 & 0 \\
$\varphi_{3}$ & 0 & 1 & 0 & 0 & 1 \\
$\varphi_{4}$ & 0 & 1 & 1 & 1 & 1 \\
$\varphi_{5}$ & 1 & 0 & 0 & 1 & 0 \\
$\varphi_{6}$ & 1 & 0 & 1 & 0 & 0 \\
$\varphi_{7}$ & 1 & 1 & 0 & 1 & 1 \\
$\varphi_{8}$ & 1 & 1 & 1 & 0 & 1
\end{tabular}

TABLE VI

Factor systems $m: Q \times Q \rightarrow C_{2}$, corresponding to operator homomorphisms of Table V (in additive notation). Each system $m$ is presented as the matrix with the element $m\left(q_{1}, q_{2}\right)$ in the $q_{1}$-th row and $q_{2}$-th column. Trivial factors $m(E, q)=m(q, E)=0, q \in D_{2}$, are omitted. Rows and columns of matrices of $m$ 's are labelled consecutively by $u_{x}, u_{y}, u_{z}$. The second cohomology group $H^{2}\left(D_{2}, C_{2}\right)$ coincides with the set $\left\{m_{1}, \ldots, m_{8}\right\}$ of factor systems of this table with the pointwise matrix addition modulo 2 as the group multiplication.

$$
\begin{array}{ll}
m_{1}=\left(\begin{array}{lll}
0 & 0 & 0 \\
0 & 0 & 0 \\
0 & 0 & 0
\end{array}\right) & m_{2}=\left(\begin{array}{lll}
0 & 0 & 0 \\
1 & 0 & 1 \\
1 & 0 & 1
\end{array}\right) \\
m_{3}=\left(\begin{array}{lll}
0 & 0 & 0 \\
0 & 1 & 1 \\
0 & 1 & 1
\end{array}\right) & m_{4}=\left(\begin{array}{lll}
0 & 0 & 0 \\
1 & 1 & 0 \\
1 & 1 & 0
\end{array}\right) \\
m_{5}=\left(\begin{array}{lll}
1 & 0 & 1 \\
0 & 0 & 0 \\
1 & 0 & 1
\end{array}\right) & m_{6}=\left(\begin{array}{lll}
1 & 0 & 1 \\
1 & 0 & 1 \\
0 & 0 & 0
\end{array}\right) \\
m_{7}=\left(\begin{array}{lll}
1 & 0 & 1 \\
0 & 1 & 1 \\
1 & 1 & 0
\end{array}\right) & m_{8}=\left(\begin{array}{lll}
1 & 0 & 1 \\
1 & 1 & 0 \\
0 & 1 & 1
\end{array}\right)
\end{array}
$$


Each cocycle $m$ defines an extension $G$ through the Seitz formula. In order to determine equivalency classes, we proceed to evaluate two-coboundaries, which are associated with crossed homomorphisms.

\section{Crossed homomorphisms and the second cohomology group}

The group

$$
Z_{\Delta \circ M}^{1}(F, T) \cong T^{X}=\{\gamma: X \rightarrow T\}
$$

of all crossed homomorphisms from $F$ to $T$ can be identified with the manifold of all mappings from the alphabet $X$ to $T$. For the case of $T=C_{2}$, we obtain four crossed homomorphisms $\gamma_{1}, \ldots, \gamma_{4}$, listed in Table VII. Using Eq. (12), it is easy

\section{TABLE VII}

The group $Z_{\Delta \circ M}^{1}\left(F, C_{2}\right)$ of all crossed homomorphisms (in additive notation for $C_{2}$ ).

\begin{tabular}{c|c|c}
\hline \hline & $x_{1}$ & $x_{2}$ \\
\hline$\gamma_{1}$ & 0 & 0 \\
$\gamma_{2}$ & 0 & 1 \\
$\gamma_{3}$ & 1 & 0 \\
$\gamma_{4}$ & 1 & 1
\end{tabular}

to evaluate that all restrictions $\left.\gamma\right|_{R}=i \circ \gamma$ of these mappings to the subgroup $R \triangleleft F$ vanish identically, i.e. that

$$
\left.\left.Z_{\Delta \circ M}^{1}\left(F, C_{2}\right)\right|_{R} \equiv Z_{M}^{1}\left(F, C_{2}\right)\right|_{R}=\{0\} .
$$

Thus the group of restrictions of crossed homomorphisms is in our case trivial (the action $\Delta$ in this case is trivial).

By virtue of Mac Lanc theorem, the second cohomology group is equal to that of operator homomorplisms, i.e.

$$
H^{2}\left(D_{2}, C_{2}\right)=\operatorname{Hom}_{F}\left(R, C_{2}\right)=\left\{m_{1}, \ldots, m_{8}\right\} .
$$

Elements of the second cohomology group can be identified with factor systems of Table VI, with the group multiplication defined as addition of corresponding matrices modulo 2 . It is an elementary Abelian group, with the unit element $m_{1}$, and all other elements of order 2.

\section{Classes of extensions of $D_{2}$ by $C_{2}$}

Each element $m \in H^{2}\left(D_{2}, C_{2}\right)$ yields the corresponding extension $G$ of $D_{2}$ by $C_{2}$, determined by the Seitz formula

$$
\left\langle t_{1}, q_{1}\right\rangle\left\langle t_{2}, q_{2}\right\rangle=\left\langle t_{1}+q_{1} \iota_{2}+m\left(q_{1}, q_{2}\right), q_{1} q_{2}\right\rangle
$$

for multiplication in $G$. There are thus 8 classes of non-equivalent extensions, which are listed in Table VIII. The trivial factor $m_{1}$ yields the elementary Abelian group 


\section{TABLE VIII}

Extension of $D_{2}$ by $C_{2}$.

\begin{tabular}{l|c|c|c|l}
\hline \hline \multirow{2}{*}{ Factor system } & \multicolumn{3}{|c|}{ Order of element } & \multirow{2}{*}{ Isomorphic group } \\
\cline { 2 - 4 } & $u_{x}$ & $u_{y}$ & $u_{z}$ & \\
\hline$m_{1}$ & 2 & 2 & 2 & $C_{2} \times C_{2} \times C_{2} \cong D_{2 h}$ \\
$m_{2}$ & 2 & 2 & 4 & $D_{4}^{z}$ \\
$m_{3}$ & 2 & 4 & 4 & $C_{2}^{x} \times C_{4}$ \\
$m_{4}$ & 2 & 4 & 2 & $D_{4}^{y}$ \\
$m_{5}$ & 4 & 2 & 4 & $C_{2}^{y} \times C_{4}$ \\
$m_{6}$ & 4 & 2 & 2 & $D_{4}^{x}$ \\
$m_{7}$ & 4 & 4 & 2 & $C_{2}^{z} \times C_{4}$ \\
$m_{8}$ & 4 & 4 & 4 & $D_{2}^{\prime}$
\end{tabular}

of order 8 , i.e.

$$
G_{1}=C_{2} \times C_{2} \times C_{2} \cong D_{2 h}
$$

with all non-trivial elements of order 2 . It is isomorphic with the crystallographic point group $D_{2 h}$. It also ariscs in quantum field thcory in the context of CPT theorem [10], as the simplest combination of discrete space and time inversions with an internal symmetry of charge conjugation. We like to mention here that, despite of the simple structure (26) of the group $G_{1}$, the appropriate Wigner-Racah calculus suffers from some puzzles concerning proper conventions of phases (cf. Chatterjee and Buckmaster [17] and references therein).

Factor systems $m_{3}, m_{5}$ and $m_{7}$ yield extensions isomorphic with the Abelian group $C_{2} \times C_{4}$. They are mutually isomorphic, but not equivalent extensions. The order of an element of the form $\left\langle\iota, u_{\alpha}\right\rangle, \iota \in C_{2}$, is either 2 for a single $\alpha=x, y, z$, or 4 for the two other $\alpha$ 's. These extensions differ mutually by the distinguished element $u_{\alpha} \in D_{2}$ associated with the twofold clement of $G$. It is denoted in Table VIII by the superscript $\alpha$ at the symbol of the isomorphic group.

Factor systems $m_{6}, m_{4}$, and $m_{2}$ correspond to dihedral groups $D_{4}$ with the fourfold axis associated respectively with $x, y$, and $z$. These extensions are thus non-Abelian groups, each with two fourfold elements $\left\langle t, u_{\alpha}\right\rangle, t \in C_{2}$, $\alpha$ fixed.

The factor system $m_{8}$ yields the double dihedral group $D_{2}^{\prime}$ with the thrce twofold axes $u_{x}, u_{y}$, and $u_{z}$ transformed into pairs $\left\{\left\langle t, u_{\alpha}\right\rangle \mid t \in C_{2}\right\}$ of fourfold elements, written usually in a form $\left\{u_{\alpha}, \bar{u}_{\alpha}\right\}, \alpha=x, y, z$, with

$$
\bar{u}_{\alpha}=\bar{E} u_{\alpha}
$$

where

$$
\bar{E}=\langle 1, E\rangle
$$

is interpreted as the rotation of the angle $2 \pi$ (around any axis) for spinors [18]. The double dihedral group $D_{2}^{\prime}$ can be readily identified with the group of quaternions, playing an important role in description of rotations of solid bodies (cf. 
Altmann [19] for a fascinating history of puzzles accompanying the use of quaternions).

It is worthwhile to observe that various extensions of $D_{2}$ by $C_{2}$ are non-isomorphic. There are four isomorphic classes of extensions. Two of them, namely $G_{1}$ and $\left\{G_{3}, G_{5}, G_{7}\right\}$, are Abelian, and the two other, $G_{8}$ and $\left\{G_{6}, G_{4}, G_{2}\right\}$ are non-Abelian. These isomorphic classes can be nicely reflected in the isomorphism betwcen the second cohomology group $H^{2}\left(D_{2}, C_{2}\right)$, and the point group $D_{2 h}$, as given in Table IX. The table shows that extcnsions within an isomorphic class can be labelled by indices $\alpha=x, y, z$ of the Cartesian coordinate system.

TABLE IX

The isomorphism between the point group $D_{2 h}$ and the second cohomology group $H^{2}\left(D_{2}, C_{2}\right)$. Vertical lines separate isomorphic classes of extensions of $D_{2}$ by $C_{2}$.

\begin{tabular}{c||c|c|c|c}
\hline$D_{2 h}$ & $E$ & $\sigma_{x} \sigma_{y} \sigma_{z}$ & $I$ & $u_{x} u_{y} u_{z}$ \\
\hline$H^{2}\left(D_{2}, C_{2}\right)$ & $m_{1}$ & $m_{6} m_{4} m_{2}$ & $m_{8}$ & $m_{3} m_{5} m_{7}$ \\
Isomorphic class & $C_{2} \times C_{2} \times C_{2}$ & $C_{2} \times C_{4}$ & $D_{2}^{\prime}$ & $D_{4}$
\end{tabular}

Isomorphic classes differ by the number of fourfold elements: $0,4,6$, and 2 for $G_{1},\left\{G_{3}, G_{5}, G_{7}\right\}, G_{8}$, and $\left\{G_{6}, G_{4}, G_{2}\right\}$, respectively. Such a doubling, or, more generally, multiplication of order of the element $q \in Q$ in the coset $T g_{q}$ of the cxtension $G$ has a known crystallographic interpretation in terms of scrcw axes or glide planes with the associated fractional translations.

\section{A comparison of Mac Lane method with an immediate application of cohomology}

Now we have demonsirated the application of Mac Lane method of classification and construction of all extcnsions of the group $D_{2}$ by $C_{2}$. In this section we make some comparison of this method with an immediate adaptation of cohomology to this case.

The colhomology theory starts with the group

$$
C^{2}\left(D_{2}, C_{2}\right)=\left\{f: D_{2} \times D_{2} \rightarrow C_{2}\right\}
$$

of all two-cochains. The order of this group is

$$
\left|C^{2}\left(D_{2}, C_{2}\right)\right|=2^{4 \cdot 4}=65536 .
$$

Each two-cochain $f \in C^{2}\left(D_{2}, C_{2}\right)$ should salisfy

$$
\left|D_{2}\right|^{3}=4^{3}=64
$$

associativity conditions

$$
\left(\delta^{2} f\right)\left(q_{1}, q_{2}, q_{3}\right)=0, \quad\left(q_{1}, q_{2}, q_{3}\right) \in D_{2}^{3},
$$

where $\delta^{2}$ is the two-coboundary operator. We observe that the associated numerical problem is rather large even in such a simple case. It increases exponentially with the increase either the active group $Q$ or the passive group $T$. 
After performing such a calculation one arrives at the group

$$
Z^{2}\left(D_{2}, C_{2}\right)=\left\{f \in C^{2}\left(D_{2}, C_{2}\right) \mid \delta^{2} f=0\right\}
$$

of all two-cocycles. The group classifies all extensions since each $f \in Z^{2}\left(D_{2}, C_{2}\right)$ serves as a distinct factor system. All these extensions are different, but some of them can be gauge equivalent. We thus need to find the group

$$
B^{2}\left(D_{2}, C_{2}\right)=\left\{\delta^{1} c \mid c \in C^{1}\left(D_{2}, C_{2}\right)\right\}=\operatorname{Im} \delta^{1}
$$

of all two-coboundaries, which is a normal subgroup in $Z^{2}\left(D_{2}, C_{2}\right)$. By the definition (34), it can be performed by an application of the one-coboundary operator $\delta^{1}: C^{1}\left(D_{2}, C_{2}\right) \rightarrow C^{2}\left(D_{2}, C_{2}\right)$ to the group

$$
C^{1}\left(D_{2}, C_{2}\right)=\left\{c: D_{2} \rightarrow C_{2}\right\}
$$

of all one-cochains $c . C^{1}\left(D_{2}, C_{2}\right)$ is the group of gauge transformations. Each gauge transformation $c$ yields an equivalent extension.

TABLE X

The gauge group $C^{1}\left(D_{2}, C_{2}\right)$. Each entry is a gauge $c: D_{2} \rightarrow C_{2}$. Each column is a coset of the gauge group $C^{1}\left(D_{2}, C_{2}\right)$ with respect to the kernel Ker $\delta^{1}=Z^{1}\left(D_{2}, C_{2}\right)$ (the group of one-cocycles).

\begin{tabular}{cccc|cccc|cccc|cccc}
\hline \hline$E$ & $u_{x}$ & $u_{y}$ & $u_{z}$ & $E$ & $u_{x}$ & $u_{y}$ & $u_{z}$ & $E$ & $u_{x}$ & $u_{y}$ & $u_{z}$ & $E$ & $u_{x}$ & $u_{y}$ & $u_{z}$ \\
\hline 0 & 0 & 0 & 0 & 0 & 0 & 0 & 1 & 1 & 0 & 0 & 0 & 1 & 0 & 0 & 1 \\
0 & 0 & 1 & 1 & 0 & 0 & 1 & 0 & 1 & 0 & 1 & 1 & 1 & 0 & 1 & 0 \\
0 & 1 & 0 & 1 & 0 & 1 & 0 & 0 & 1 & 1 & 0 & 1 & 1 & 1 & 0 & 0 \\
0 & 1 & 1 & 0 & 0 & 1 & 1 & 1 & 1 & 1 & 1 & 0 & 1 & 1 & 1 & 1
\end{tabular}

The order of the gauge group is

$$
\left|C^{1}\left(D_{2}, C_{2}\right)\right|=2^{4}=16
$$

(cf. Table $\mathrm{X}$ ). The two-coboundary $\delta^{1} c$ associated with a gauge $c \in C^{1}\left(D_{2}, C_{2}\right)$ is given by

$$
\left(\delta^{1} c\right)\left(q_{1}, q_{2}\right)=c\left(q_{1}\right)+c\left(q_{2}\right)-c\left(q_{1} q_{2}\right)
$$

(since the action of $D_{2}$ in $C_{2}$ is trivial). The kernel

$$
Z^{1}\left(D_{2}, C_{2}\right)=\operatorname{Ker} \delta^{1}=\left\{c \in C^{1}\left(D_{2}, C_{2}\right) \mid \delta^{1} c=0\right\}
$$

is a subgroup of $C^{1}\left(D_{2}, C_{2}\right)$, given in the first column of Table X. It consists of all such gauges which do not change any factor system. In our case, when the action of $D_{2}$ on $C_{2}$ is trivial, each such a gauge defines a representation of the active group $D_{2}$, valued in the passive group $C_{2}$. 
TABLE XI

The group $B^{2}\left(D_{2}, C_{2}\right)$ of all two-coboundaries. Each two-coboundary defines a trivial factor system. Rows and columns of each matrix are labelled consecutively by $q=E, u_{x}, u_{y}, u_{z}$.

$$
\begin{aligned}
& b_{1}=\left(\begin{array}{llll}
0 & 0 & 0 & 0 \\
0 & 0 & 0 & 0 \\
0 & 0 & 0 & 0 \\
0 & 0 & 0 & 0
\end{array}\right) b_{2}=\left(\begin{array}{llll}
0 & 0 & 0 & 0 \\
0 & 0 & 1 & 1 \\
0 & 1 & 0 & 1 \\
0 & 1 & 1 & 0
\end{array}\right) \\
& \delta_{3}=\left(\begin{array}{llll}
1 & 1 & 1 & 1 \\
1 & 1 & 0 & 0 \\
1 & 0 & 1 & 0 \\
1 & 0 & 0 & 1
\end{array}\right) \quad b_{4}=\left(\begin{array}{llll}
1 & 1 & 1 & 1 \\
1 & 1 & 1 & 1 \\
1 & 1 & 1 & 1 \\
1 & 1 & 1 & 1
\end{array}\right)
\end{aligned}
$$

Equation (37) and Table $X$ yield the group $B^{2}\left(D_{2}, C_{2}\right)$ of all two-coboundaries (one has to apply Eq. (37) to a single gauge $c$ for each column of Table $X$ ). This group is given in Table XI. We observe that the two-coboundaries $b_{1}$ and $b_{2}$ satisfy the defining condition

$$
m(E, q)=m(q, E)=0, q \in D_{2}
$$

for a normalized factor system, whereas $b_{3}$ and $b_{4}$ are unnormalized.

Thus the group $B^{2}\left(D_{2}, C_{2}\right)$ can be easily evaluated merely from the cohomological definition. It is not the case for the group $Z^{2}\left(D_{2}, C_{2}\right)$ of all two-cocyclcs, where much more numerical effort is needed. Using the fact that the order of the second cohomoly group is 8 , we oblain

$$
\left|Z^{2}\left(D_{2}, C_{2}\right)\right|=\left|B^{2}\left(D_{2}, C_{2}\right)\right|\left|I^{2}\left(D_{2}, C_{2}\right)\right|=4 \cdot 8=32 \text {. }
$$

Table XI implies that the factor systems of Table VI are not unique, but they are given only modulo the group $B^{2}\left(D_{2}, C_{2}\right)$ of all two-coboundaries. Even if we restrict ourselves to normalized factor systems (39), still each $m$ of Table VI can be substituted by a gaugc-cquivalent factor system

$$
m^{\prime}=m+b_{2} \text {. }
$$

Thus, e.g. the factor system $m_{8}$ of Table VI

$$
m_{8}=\left(\begin{array}{ccc}
1 & 0 & 1 \\
1 & 1 & 0 \\
0 & 1 & 1
\end{array}\right)
$$

is gauge-equivalent to

$$
m_{8}^{\prime}=\left(\begin{array}{ccc}
1 & 1 & 0 \\
0 & 1 & 1 \\
1 & 0 & 1
\end{array}\right) .
$$

In terms of the extension $G_{8}$, i.e. the double group $D_{2}^{\prime}$, it means that the relations

$$
u_{x} u_{y}=u_{z}
$$


and

$$
u_{x} u_{y}=\bar{u}_{z} \equiv \bar{E} u_{z}
$$

for the double group $D_{2}^{\prime}$ are gauge-equivalent (cf. [20]). This arbitrariness is nicely reflected in the fibre structure of the extension $G_{8}$, where $\left\{u_{z}, \bar{u}_{z}\right\} \in D_{2}^{\prime}$ forms the fiber over $u_{z} \in D_{2}$, and each elcment of this fiber is equally good as the candidate for $\left\langle 0, u_{x}\right\rangle\left\langle 0, u_{y}\right\rangle$.

\section{References}

[1] T. Lulek, Acla Phys. Pol. A 82, 377 (1992).

[2] R.E. Prange, S.M. Girvin (Eds.), The Quanlum IIall Effecl, Springer-Verlag, Berlin 1981.

[3] M.V. Berry, Proc. R. Soc. Lond. A 392, 45 (1984).

[4] B. Simon, Phys. Rev. Lell. 51, 2167 (1983).

[5] Y. Alaronov, J. Anandan, Phys. Rev. Lell. 58, 1593 (1987).

[6] D.J. Thouless, M. Kohmoto, M.P. Nightingale, M. den Nijs, Phys. Rev. Lell. 49, 405 (1982).

[7] M. Kohmoto, Ann. Pliys. 160, 343 (1985).

[8] D.J. Thouless, in Ref. [2], p. 101.

[9] F. Wilczek, Phys. Rev. Lell. 48, 1144 (1982).

[10] R.F. Streater, A.S. Wightman, PCT, Spin and Statistics and All That, Benjamin, New York 1964.

[11] M. Mucha, in: Symmetry and Struclural Properties of Condensed Maller, Eds. W. Florek. T. Lulek, M. Mucha, World Sci., Singapore 1991, p. 19.

[12] M. Kuźma, in Ref. [11], p. 379.

[13] B. Lulek, T. Lulek, M. Kużma, Acla Phys. Pol. B 22, 287 (1991).

[14] G. James, A. Kerber, The Representation Theory of the Symmetric Group, Encycl. Math. Appl., Vol. 16, Addison-Wesley, Reading 1981.

[15] A. Kerber, B. Lulek, T. Lulek, in Ref. [11], p. 3.

[16] T. Lulek, J. Biel, Acla Magnetica 1, 99 (1984), A. Mickiewicz Univ. Publ., Poznań 1984.

[17] R. Chatterjee, II.A. Buckınaster, Ref. [11], p. 230.

[18] S.L. Altmann, Rolations, Quaternions, and Double Groups, Clarendon, Oxford 1986.

[19] S.L. Altmann, Math. Mag. 62, 291 (1989).

[20] G.W. Koster, J.O. Dimmock, R.G. Wheeler, M. Statz, Properties of the Thirty-Two Point Groups, MIT Press, Cambridge, Massachusetts 1963. 